\title{
Frauen in Führungspositionen - Kommt die „europäische“ Quote?
}

\author{
Veranstaltung der Regionalgruppe Brüssel im djb und der Vertretung des Freistaates Bayern bei \\ der Europäischen Union am 27. März 2013 in der Bayerischen EU-Vertretung in Brüssel
}

Am 27. März 2013 fand in den schönen Räumlichkeiten der Bayerischen EU-Vertretung in Brüssel eine gemeinsam von der djb-Regionalgruppe Brüssel und der Vertretung des Freistaates Bayern bei der EU organisierte Mittagsveranstaltung zum Thema „Kommt die ‘europäische’ Quote?“ statt. Die gemeinsame Veranstaltung war schon lange geplant, der Zeitpunkt stellte sich aber als besonders günstig heraus, da die Beratungen des am 14. November 2012 von der EU-Kommission verabschiedeten Quoten-Richtlinienvorschlags sowohl im Europäischen Parlament als auch im Ministerrat an Fahrt aufgenommen hatten. Zudem trug zur Aktualität der Veranstaltung - und anschließenden Lebhaftigkeit der Diskussion - auch bei, dass die Bundesregierung sich kurz vorher unter Hinweis auf den Subsidiaritätsgrundsatz offiziell gegen den Richtlinienvorschlag von Viviane Reding positioniert hatte.

Nach der Begrüßung durch die Leiterin der Bayerischen Vertretung, Dr. Angelika Schlunck, und die Vorsitzende der RG Brüssel im djb, Dr. Annette Matthias, hielt djb-Präsidentin Ramona Pisal ein engagiertes Impulsreferat pro Quote, in dem sie unter anderem auch das 2009 ins Leben gerufene djb-Projekt „Aktionärinnen fordern Gleichberechtigung “ vorstellte. ${ }^{1}$ Das sich anschließende Panel war mit viel Sach- und Fachverstand und zudem noch „geschlechtergerecht“ je zur Hälfte mit Frauen und Männern besetzt: Neben Ramona Pisal diskutierten unter der Leitung von Dr. Detlef Fechtner, Börsenzeitung, Marije Cornelissen MdEP, Mitglied im Ausschuss für die Rechte der Frau und Gleichstellung der Geschlechter im Europäischen Parlament und Berichterstatterin für den Richtlinienvorschlag im Beschäftigungsausschuss, Andreas Stein, zuständiger Abteilungsleiter in der Generaldirektion Justiz der EU-Kommission, Mark Kamperhoff, der Leiter des BMFSFJ-Arbeitsbereichs in der deutschen EU-Vertretung, und Christine Bruchmann, Geschäftsführende Gesellschafterin der Moritz Fürst GmbH \& Co. KG. Wenn auch die Meinungen zur Notwendigkeit einer verbindlichen Quote

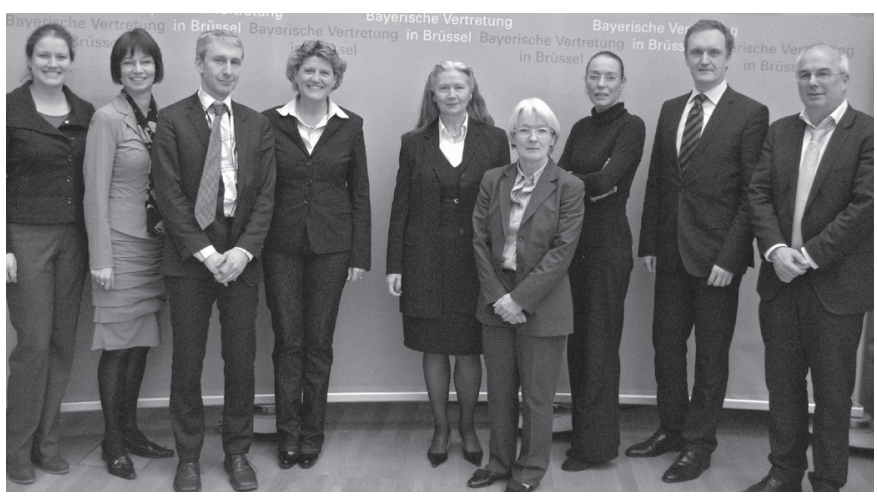

$\Delta$ (v.l.n.r.) Patricia Hamel, Vertretung des Freistaates Bayern bei der Europäischen Union, Dr. Angelika Schlunck, Leiterin der Vertretung des Freistaates Bayern bei der EU, Andreas Stein, Europäische Kommission, GD Justiz, Abteilungsleiter Gleichstellungsrecht, Christine Bruchmann, Geschäftsführende Gesellschafterin Moritz Fürst GmbH \& Co. KG, Ramona Pisal, djb-Präsidentin, Dr. Annette Matthias, Vorsitzende der Regionalgruppe Brüssel im djb, Marije Cornelissen, MdEP, Mitglied im Ausschuss für die Rechte der Frau und Gleichstellung der Geschlechter, Mark Kamperhoff, Ständige Vertretung der Bundesrepublik Deutschland bei der EU, Leiter des Arbeitsbereichs Familie, Senioren, Frauen und Jugend, Dr. Detlef Fechtner, Börsenzeitung. (Foto: Margarete Hofmann, djb)

durchaus geteilt waren, bestand allseits Einverständnis, dass es höchste Zeit und ein Gebot nicht nur der Gerechtigkeit, sondern auch der wirtschaftlichen Vernunft ist, dass Frauen endlich auch in Führungspositionen der Wirtschaft angemessen vertreten sind.

Das zahlreich erschienene Publikum beteiligte sich lebhaft an der Diskussion und setzte den Meinungsaustausch anschließend bei Kaffee und Kuchen fort.

(Margarete Hofmann, djb-Vizepräsidentin)

1 Der Vortrag von djb-Präsidentin Ramona Pisal ist im Folgenden hier abgedruckt.

\section{Von nichts kommt nichts - Europa braucht die Frauenquote'}

\section{Ramona Pisal \\ Präsidentin des djb, Berlin}

\footnotetext{
Sehr geehrte Frau Dr. Schlunck, sehr geehrte Frau Dr. Matthias, sehr geehrte Damen und Herren Abgeordnete des Europäischen Parlaments, sehr geehrte Damen und Herren auf dem Podium und im $\mathrm{Pu}-$ blikum, liebe Kolleginnen im Deutschen Juristinnenbund,
}

es freut mich sehr, dass ich heute Mittag auf Einladung der Bayerischen Vertretung bei der Europäischen Union und der Regionalgruppe Brüssel des djb hier Ihr Gast sein darf. Das Thema „Frauen in Führungspositionen“ beschäftigt den djb seit vielen Jahren. Es ist mir darum eine besondere Ehre, Ihnen

1 Rede gehalten bei der gemeinsamen Veranstaltung Frauen in Führungspositionen - Kommt die „europäische“ Quote? der Vertretung des Freistaates Bayern bei der Europäischen Union und der Regionalgruppe Brüssel im djb am 27. März 2013 in der Bayerischen EU-Vertretung in Brüssel. 
unser Projekt „Aktionärinnen fordern Gleichberechtigung“ zum Auftakt unserer Diskussion kurz vorstellen zu dürfen.

Wie Sie alle wissen, steht Deutschland im internationalen Vergleich nicht gut da. Schauen wir auf die 200 größten Unternehmen in Deutschland, ${ }^{2}$ so finden wir dort nur vier Prozent Frauen in Vorstandspositionen, nur 13 Prozent der Aufsichtsräte sind weiblich. Und dieser magere Befund ist im Vergleich zu den Vorjahren schon ein Fortschritt!

Mehr als ein halbes Jahrhundert nach Inkrafttreten unserer Verfassung steht der ebenso simple wie selbstverständliche Satz „Männer und Frauen sind gleichberechtigt" nur auf dem Papier, was die deutsche Wirtschaft anlangt. Dem wollte die rot-grüne Koalition mit einem Gleichstellungsgesetz für die Privatwirtschaft vor 13 Jahren eigentlich ein Ende machen. Aber die Wirtschaftslobby war stärker. Sie versprach 2001, das Problem selbst zu lösen und mehr Frauen in Führungsverantwortung zu bringen. Das nachfolgende Jahrzehnt hat dieses Versprechen als nicht ernst gemeint entlarvt. Im Jahr 2009 gab es genau eine Frau im Vorstand eines DAX-Unternehmens. Von den Aufsichtsräten waren nur zehn Prozent weiblich, wobei nur eine von vier Frauen von der Seite der Anteilseigner in den Aufsichtsrat entsandt wurde, also nur 2,5 Prozent.

Das wollten wir Juristinnen nicht länger hinnehmen. Das deutsche Aktienrecht gibt den Aktionärinnen und Aktionären das Recht, auf den jährlichen Hauptversammlungen Fragen an Aufsichtsrat und Vorstand zu richten, die dann auch beantwortet werden müssen. Dieses Instrument ist das Kernstück des Projektes, das der djb aktuell zum vierten Mal in Folge durchführt. Das Projekt wird vom Bundesministerium für $\mathrm{Fa}-$ milie, Senioren, Frauen und Jugend gefördert.

Seit 2010 besuchen wir alle 30 DAX-Unternehmen und 46 weitere große, börsennotierte Aktiengesellschaften. Für die Ausübung des Stimm- und damit des Fragerechtes genügt eine einzige Aktie. Als Aktionärin oder Vertreterin fragen wir danach, warum so wenige Frauen im Aufsichtsrat oder im Vorstand des Unternehmens sind, ob die Unternehmen konkrete Ziele verfolgen für die Erhöhung des Frauenanteils in diesen Gremien und in den anderen Führungsebenen, welche Strategien sie konkret verfolgen, und in diesem Jahr fragen wir auch danach, ob die Unternehmen schon einmal der Frage nachgegangen sind, ob die Erhöhung des Frauenanteils in den Führungsetagen in ihrem Interesse liegen könnte, unter anderem aus wirtschaftlichen Gründen.

Die Antworten, die wir bekommen, stimmen uns nicht sonderlich optimistisch, dass wir in den nächsten Jahren deutlich mehr Frauen in Führungspositionen in den Topetagen der deutschen Wirtschaft antreffen werden. Zahlreiche Unternehmen suchen ihre Aufsichtsräte ausschließlich oder überwiegend über eigene Netzwerke. Sie nehmen weder Personalberatung in Anspruch noch greifen sie auf Datenbanken zurück, wie sie zum Beispiel der Verband der Unternehmerinnen mit inzwischen ca. 500 für Aufsichtsratsmandate qualifizierten Frauen führt. Von den Unternehmen, die Personalberater/innen einschalten, bitten nur ein Drittel gezielt auch um die Benennung von Frauen.
Zur Veranschaulichung einige Eckdaten: ${ }^{3}$

Nur jede fünfte Neubestellung von Aufsichtsräten bei den 30 DAX-Werten erfolgte 2012 von Arbeitgeberseite, zwei Drittel der Frauen werden von den Arbeitnehmern entsandt. Konkret heißt das: Bei den 30 umsatzstärksten deutschen Unternehmen, den sogenannten DAX-Werten, sind 213 Männer, aber nur 39 Frauen in den Aufsichtsräten auf Anteilseignerseite und es gibt nur eine Vorsitzende eines Aufsichtsrates (Dr. Simone Bagel-Trah bei Henkel, die zur Eigentümerfamilie gehört). Im letzten Jahr sind 58 Männer gewählt worden, aber nur 16 Frauen, bezogen auf die 30 DAX-Werte.

Daran wird sich so schnell auch nichts ändern, wenn die Unternehmen weiterhin nur in geschlossenen Netzwerken nach Kandidatinnen und Kandidaten für die Aufsichtsräte suchen.

Bei den Vorständen sieht es nicht besser aus. Nur sechs der 30 DAX-Werte, jedes fünfte Unternehmen, also 20 Prozent, entwickelt aktuell Frauen für Vorstandspositionen. Von den 193 Vorständen sind nur 15 Frauen, verteilt auf 13 Unternehmen, keine Frau ist Vorstandsvorsitzende. 17 Unternehmen im DAX haben keine Frau im Vorstand. Unter den 41 Neubestellungen waren nur sieben Frauen, dies entspricht 19 Prozent.

Das ist ein durchschnittlicher Anstieg von 2,3 Prozent im Jahr.

Mit weiteren Zahlen will ich Sie nicht behelligen. Die Einzelheiten können Sie bei Interesse gerne unserer Auswertung entnehmen, die wir zuletzt im November 2012 veröffentlicht haben.

Ganz generell gilt: Der Anteil der Frauen an der Belegschaft bildet sich in den Führungsebenen nicht ab. Er sinkt zudem mit jeder Beförderungsstufe. Und für alle Unternehmen lässt sich sagen: Je höher die Führungsebene, desto niedriger der Frauenanteil. Insoweit gibt es keine signifikanten Veränderungen gegenüber dem Vorjahr.

Ihr Versprechen haben die 30 DAX-Unternehmen mit einer sogenannten Selbstverpflichtung bekräftigt. Diese bezieht sich jedoch nicht auf Vorstand und Aufsichtsrat, sondern auf die Führungsebenen darunter, und zwar in summa.

Unser Projekt „Aktionärinnen fordern Gleichberechtigung “ bindet sehr viel ehren- und auch hauptamtliche Arbeit. Warum machen wir das?

Durch unsere Fragen in der Hauptversammlung wollen wir die Verantwortlichen in Vorstand und Aufsichtsrat für das Thema sensibilisieren und Bewusstsein schaffen.

Für den offensichtlichen Missstand wollen wir eine öffentliche Rechtfertigung und Erklärung einfordern nach dem Motto: name and shame.

Wir wollen ein Umdenken anstoßen und ein Umsteuern bewirken, Öffentlichkeit herstellen und Transparenz in die Aus-

2 Holst, Elke/Schimeta, Julia, Frauenanteil in Topgremien großer Unternehmen in Deutschland nimmt geringfügig zu - DAX-30Unternehmen mit größerer Dynamik, in: DIW Berlin - Deutsches Institut für Wirtschaftsforschung e.V. (Hrsg.), DIW-Wochenbericht 3-2013. Managerinnen-Barometer 2013, 80. Jg. Januar 2013, S. 3 ff., online erhältlich unter <http://www.diw.de/documents/ publikationen/73/diw_01.c.414308.de/13-3.pdf〉 (Zugriff: 29.5.2013). 3 Stand: 31.12.2012, Recherche des djb. 
wahlprozesse bringen. Insoweit sind wir ganz in Übereinstimmung mit dem Richtlinienvorschlag der Kommission.

Gleichermaßen wollen wir bei den Aktionärinnen und Aktionären wirken. Sie sollten ein großes Interesse daran haben, dass unternehmerische Entscheidungen abgewogen und unter Einbeziehung vielfältiger Perspektiven getroffen werden. Sie wählen die Aufsichtsräte.

Und wir wollen den Handlungsbedarf für die Politik aufzeigen, denn den gibt es, wie unser Projekt nun im vierten Jahr ganz deutlich zeigt. Die Unternehmen antworten vielfach ausweichend, verfolgen überwiegend keine konkreten Ziele, um mehr Frauen in Führungspositionen zu bringen, können keine Strategien dazu benennen und haben keine Strukturen geschaffen, die darauf angelegt sind, kontinuierlich und nachhaltig mit messbaren Fortschritten Frauen in Führungspositionen zu bringen.

Stattdessen müssen wir immer wieder hören, dass Führungskräfte ausschließlich nach ihrer Qualifikation ausgesucht werden, dass es nicht genügend qualifizierte Frauen gibt, dass die wenigen qualifizierten Frauen keine Führungsverantwortung übernehmen wollen und schließlich soll es dann immer wieder an mangelnder Vereinbarkeit von Familie und Beruf scheitern, dass wir vergeblich auf die Frauen in den Topetagen warten. Als ob Männer keine Kinder hätten, wird dieses Problem im Zusammenhang mit männlichen Karrieren niemals bemüht. Dabei hätten wir eine Frauenquote von 40 Prozent ohne Weiteres längst erreicht, wenn sich schon nur der Anteil der kinderlosen Akademikerinnen an den Führungskräften abbilden würde, denn dieser Anteil liegt bei 40 Prozent.

All diese Erklärungen sind aus unserer Sicht nichts als Vorwände. Sie sollen verschleiern, dass Frauen ganz oben nicht anzutreffen sind, weil Männer dort lieber unter sich bleiben, Macht und Einfluss nicht teilen wollen, neben der unausweichlichen männlichen jedenfalls die zusätzliche weibliche Konkurrenz lieber ausschalten. Freiwillig wird nicht geteilt.

Die marginalen Veränderungen der letzten vier Jahre sind ausschließlich dem öffentlichen Druck geschuldet und dem Bemühen, durch halbherzige Aktionen den Gesetzgeber ruhig zu stellen. Sobald diese Gefahr gebannt ist, werden aus 15 Vorstandsfrauen wieder eine und schließlich keine werden, davon bin ich überzeugt. Die Erfahrung eines halben Jahrhunderts spricht dafür, dass ich mit dieser düsteren Prognose nicht falsch liege. Wir brauchen gesetzgeberische Maßnahmen, damit der Impuls nicht versandet und der zaghafte Fortschritt nachhaltig weiterentwickelt wird. Das hat auch die Politik erkannt.

Bereits im Oktober 2010 hat die Bundestagsfraktion Bündnis 90/Die Grünen einen Gesetzentwurf vorgelegt, der 40 Prozent Frauen für die Aufsichtsräte und Sanktionen bei Nichterreichen dieses Zieles vorsieht. Die SPD und die Linke gehen mit ihren Entwürfen darüber hinaus. Nordrhein-Westfalen hat einen Entwurf lanciert, die Justizministerkonferenz hat sich für eine Quote per Gesetz ausgesprochen, die CDU hätte sich mit einer flexiblen Quote anfreunden können, und schließlich hat ein Gesetzentwurf aus Hamburg mit Unterstützung der Länder Sachsen-Anhalt und Saarland den Bundesrat passiert; er liegt nun dem Rechtsausschuss des Bundestages vor. All das zeigt einen breiten gesellschaftlichen Konsens dahingehend, dem klaren Verfassungsauftrag zur tatsächlichen Gleichstellung von Frauen und Männern auch im wirklichen Leben endlich wirksam Geltung zu verschaffen. Denselben Auftrag formuliert die Grundrechtecharta in Artikel 23.

Dafür steht auch die Berliner Erklärung, ein Zusammenschluss von 12 Frauen aus sechs Verbänden und sechs Fraktionen, die sich auf eine Forderung von mindestens 30 Prozent Frauen für die Aufsichtsräte der mitbestimmten börsennotierten Unternehmen geeinigt haben. Einen derartigen Schulterschluss, überfraktionell und zwischen Politikerinnen und Verbänden, hat es in vielen Jahrzehnten zu keinem anderen Thema gegeben. Die Berliner Erklärung unterstreicht den Stellenwert, den die Diskussion um eine Frauenquote per Gesetz in der deutschen Öffentlichkeit inzwischen erreicht hat. Die Demokratie wird sich daran messen lassen müssen, wie sie mit einer Hälfte der Bevölkerung umgeht.

Und die Diskussion zieht Kreise. Vor gut einem Jahr haben Journalistinnen den Verein ProQuote Medien e.V. gegründet. Sie fordern einen Anteil von 30 Prozent Frauen in den Chefredaktionen. Aktuell sind nur zwei Prozent Chefredakteurinnen dort anzutreffen. Und erst vor wenigen Wochen gründete sich ProQuote Medizin. Wussten Sie, dass lediglich drei Lehrstühle für Gynäkologie in Deutschland mit einer Frau besetzt sind?

Im Deutschen Bundestag haben es die Initiativen zu einer verbindlichen Frauenquote derzeit sehr schwer. Die Opposition allein verfügt naturgemäß nicht über genug Stimmen, die Regierungsparteien sind sich nicht einig, der Fraktionszwang hindert die Zustimmung derer, die mit uns sind, und das Ende der Legislaturperiode ist bald erreicht. Damit droht der wertvolle Aufbruch ins Leere zu laufen. In dieser Situation brauchen wir Europa dringend und den abgewogenen Vorstoß der Kommission.

Schon immer sind von Europa wegweisende Impulse für die Gleichstellung der Frauen ausgegangen. So ist es auch beim Thema Frauen in Führungspositionen, das von der Vizepräsidentin der Kommission, Viviane Reding, mit großem Engagement und Tatkraft platziert worden ist. Man mag bedauern, dass ihr ambitionierter Entwurf für einen Richtlinienvorschlag im politischen Prozess deutliche Abstriche erfahren musste, aber so ist das eben, wenn ein Konsens verhandelt sein will. Der endgültige Vorschlag weist trotzdem in die richtige Richtung und trägt gleichzeitig den Bedenken Rechnung, dass die Unternehmen überfordert und die Nationalstaaten in ihrer Souveränität berührt sein könnten: Die Quote beschränkt sich auf Aufsichtsräte, sieht lange Übergangsfristen vor und - was wir bedauern - auch viele Ausnahmen. Die wichtige Frage der Sanktionen wird den Mitgliedstaaten überlassen. Wer selbst tätig wird, muss dem Vorschlag aus Brüssel nicht folgen. Eine moderate Lenkung in einer sehr entscheidenden Frage, sollte man meinen. Anders die Bundesregierung und mit ihr andere Staaten, darunter auch solche, von denen wir erwartet hätten, dass sie weiter sind als wir in Deutschland.

Mit wachsendem Zorn stehen wir Frauen und Männer aus der Mitte der Gesellschaft vor diesem „Nein“ und als Betrof- 
fene schämen wir uns für die Verantwortlichen, schämen wir uns an ihrer Stelle dafür, welches Bild Deutschland mit diesem „Nein“ im europäischen Kontext abgibt. Da tut es gut, zu wissen, dass es auch in der Bundesregierung andere Stimmen gibt, Frauen und Männer, die sich für die gleichberechtigte Teilhabe von Frauen in allen gesellschaftlichen Bereichen ein- setzen und unsere Forderung nach einer Quote jedenfalls für die Aufsichtsräte in der Privatwirtschaft unterstützen.

Ich bin gespannt auf die Diskussion mit Ihnen und freue mich auf Ihre Erfahrungen und den Austausch.

Haben Sie ganz herzlichen Dank für Ihre Aufmerksamkeit.

\section{Ende der Märchenstunde - Über das Scheitern der Frauenpolitik in der 17. Legislaturperiode}

\section{Katrin Lange}

Projektmitarbeiterin, Deutscher Juristinnenbund, Berlin

Am 18. April 2013 stand auf der Tagesordnung der Plenarsitzung des Deutschen Bundestages die Abstimmung über gleich mehrere Initiativen zur Einführung einer Quote in Führungsgremien. ${ }^{1}$ Nicht dass mit einer Überraschung im Abstimmungsverhalten der gewählten Vertreterinnen und Vertreter dieser Republik wirklich zu rechnen gewesen wäre, wenige Tage vor der Debatte stand spätestens die bevorstehende Ablehnung der Gesetzesanträge fest. Die Haltung der Union konnte, trotz Zugeständnissen der Opposition ${ }^{2}$, in Hinblick auf eine Zustimmung der Initiativen nicht beeinflusst werden. Um aber einem drohenden Abstimmungsdilemma zu entgehen, hatte die CDU in einer bemerkenswerten Kehrtwende ihre Reihen mit einem Kompromiss geschlossen: die Aufnahme einer festen Quote von 30 Prozent im Jahr 2020 ins anstehende Wahlprogramm. In der Folge stimmten selbst Befürworter/innen der Quote gegen die Anträge. Eine notwendige Mehrheit konnte erwartungsgemäß somit nicht erreicht werden.

Was ist nun der Stand der Dinge? Trotz des erneut gescheiterten Versuchs, eine Quote für Führungsgremien gesetzlich zu verankern, und der somit historisch verpassten Chance, gleichberechtigte Teilhabe von Frauen in der Privatwirtschaft zu sichern, bleibt der Druck auf Politik und Wirtschaft ungebrochen.

In der Berliner Erklärung ${ }^{3}$ kämpfen seit Dezember 2011 Vertreterinnen führender Frauenverbände ${ }^{4}$ und Abgeordnete aller sechs im Bundestag vertretenen Parteien gemeinsam für eine Quote von mindestens 30 Prozent Frauen für die Aufsichtsräte. Diese Allianz, überfraktionell und zwischen Politik und Zivilgesellschaft geschlossen, ist einzigartig und wird mittlerweile von knapp 23.000 Unterzeichnerinnen und Unterzeichnern mit getragen. Ausdrücklich wird sich so zu dem gemeinsamen Ziel, Frauen und Männern in allen gesellschaftlichen Bereichen gleiche Verwirklichungs- und Teilhaberechte einzuräumen, bekannt. Dies zeigt auch, welche Bedeutung die Debatte über eine gesetzliche Regelung einer Frauenquote in der Gesellschaft inzwischen eingenommen hat. In einem kurz vor der Abstimmung im Bundestag veröffentlichten offenen Brief forderten die Initiatorenverbände der Berliner Erklärung zusammen mit vier weiteren Verbänden ${ }^{5}$ alle 620 Abgeordneten des Bundestages ausdrücklich auf, für die Initiative des Bundesrats, die zur namentlichen Abstimmung stand, zu stimmen. Im Ergebnis stimmten von 598 Abgeordneten 320 mit Nein und 277 für Ja. Es gab eine Enthaltung. ${ }^{6}$ Einzig Sibylle Laurischk, Abgeordnete der FDP-Fraktion und MitInitiatorin der Berliner Erklärung, stimmte zusammen mit der Opposition für den Gesetzesantrag. ${ }^{7}$

Die CDU hat mit ihrem Nein zur Quote die letzte Möglichkeit verstreichen lassen, noch in dieser Legislaturperiode einen deutlichen Impuls hinsichtlich des Stellenwerts von Frauen in Wirtschaft und Gesellschaft zu setzen. Hervorzuheben bleibt allein, dass ihr Kompromiss einen seit Jahren andauernden parteiinternen Streit zwischen Bundesarbeitsministerin Ursula von der Leyen und Bundesfamilienministerin Kristina Schröder über die Ausgestaltung von Maßnahmen, die zur Erhöhung des Anteils von Frauen in Führungspositionen beitragen sollen, zu beenden scheint. Erstmals ist eine gesetzliche Festlegung einer festen Frauenquote für Unternehmen in der CDU überhaupt vorgesehen. Lange hat Ursula von der Leyen sich dafür eingesetzt. Letztlich half der Druck, den sie durch ihre bis dahin offen gelassene Überlegung auslöste, sich der Opposition anzuschließen und für die Quote zu stimmen.

Nun sind also beide Quotenkonzepte im Kompromiss vereint. Bis 2020 wird die parteiintern nicht unumstrittene FlexiQuote, die vorrangig auf freiwillige Selbstverpflichtung der Unternehmen setzt, verfolgt. Sollte sich kein nennenswerter Erfolg einstellen, kommt ab 2020 die feste Quote. Die Idee, auf freiwillige Selbstverpflichtungen der Wirtschaftsunternehmen zu setzen, konterkariert die Union somit in zweifacher Hinsicht. Zum einen, indem sie die Flexi-Quote selbst zum

1 Informationen zu den Gesetzesinitiativen in: Lange, Katrin, Was bisher geschah. Das Ringen um die gesetzliche Regelung einer Frauenquote in wirtschaftlichen Führungspositionen, in dieser Ausgabe S. 70.

2 Nicht nur, dass die Gesetzesinitiativen selbst Produkt eines Minimalkonsenses der verschiedenen Fraktionen sind, in einem am Vortag der Abstimmung eingereichten Änderungsantrag der Grünen wurde in allerletzter Sekunde auf den kurz zuvor geschlossenen Konsens in der Unionsfraktion eingegangen, demzufolge entsprechend für 2020 eine Quote von 30 Prozent Frauenanteil vorgesehen ist.

3 <http://www.berlinererklaerung.de/> (Zugriff: 18.4.2013).

4 Die insgesamt zehn Frauenverbände und -initiativen haben mehr als 500.000 Mitglieder und Unterstützer/innen.

5 Deutscher Ingenieurinnenbund e.V., ProQuote Medien e.V., ProQuote Medizin und Nürnberger Resolution.

6 Siegfried Kauder, Abgeordneter der CDU/CSU-Fraktion und Vorsitzender des Rechtsausschusses im Bundestag, Bruder von Volker Kauder, Vorsitzender der CDU/CSU-Fraktion.

7 <http://dip21.bundestag.de/dip21/btp/17/17234.pdf> (Zugriff: 19.4.2013). 